\title{
The nail under fungal siege in patients with type II diabetes mellitus
}

\author{
Gérald E. Piérard and Claudine Piérard-Franchimont
}

Department of Dermatopathology, University Hospital of Liège, Liège, Belgium

\section{Summary}

\begin{abstract}
Few studies have examined the prevalence of onychomycosis among diabetic patients. Given the morbidity linked to onychomycosis, and the ever-growing size of the diabetic population, a better recognition of this nail infection is welcome. To revisit the relative prevalence of dermatophyte, yeast and non-dermatophytic mould onychomycoses in diabetic adults in a prospective study using combined histomycology and cultures. Toenail clippings were collected for 3 years in 190 type II diabetic patients (136 men and 54 women) and from an age- and gender-matched group of non-diabetic subjects. All sampled nails showed clinical alterations reminiscent of onychomycosis. Histomycology and cultures were performed on each sample to distinguish onychomycosis from non-infectious onychodystrophy. Compared to non-diabetic subjects with nail alterations, diabetics showed a higher proportion of onychomycosis relative to non-fungal onychodystrophy. Diabetic men suffered more frequently from onychomycosis and onychodystrophy than diabetic women. When considering the nature of the fungal pathogens, dermatophytes predominated largely over yeast and non-dermatophytic moulds, both in diabetic and non-diabetic patients. Diabetic patients, particularly men, are at increased risk of developing onychomycosis. The morbidity linked to this disorder, and its impact on the foot status in diabetic subjects merit to be better appreciated by clinicians.
\end{abstract}

Key words: nail fungus, onychomycosis, diabetic foot.

\section{Introduction}

The endeavours made in the past to find a quick, safe and, in the long run, satisfactory solution to the problem posed by onychomycosis were not completely successful. Topical and systemic therapeutic regimens exist, but have so far failed to meet general wishful thinking. The problem is more complex in polymedicated patients and in those showing an increased propensity to develop fungal infection of the nails. ${ }^{1,2}$

The high frequency with which foot disorders occur in diabetic patients turns it into considerable public health problem. Nail alterations can be one of the problems of this condition. ${ }^{3,4}$ In the past decade or so

Correspondence: Gérald E. Piérard, Department of Dermatopathology, CHU Sart Tilman, B-4000 Liège, Belgium.

Tel.: +32 4 3662408. Fax: +3243662976.

E-mail: gerald.pierard@ulg.ac.be

Accepted for publication 8 June 2005 several studies have evaluated the prevalence of onychomycosis in these patients. ${ }^{5-16}$ Overall, clinically abnormal nails with mycological evidence of fungal disease have been reported in approximately one-thirds of diabetics. ${ }^{9}$ The highest prevalence was reported in men with type II diabetes. ${ }^{8,9,11,13}$ The overall risk ratio of diabetic patients having onychomycosis was estimated as high as 2.77 compared with age- and sexmatched non-diabetic subjects. ${ }^{9}$ In some studies, yeast infections of the nail were found to be more frequent in diabetic patients. ${ }^{16,17}$ However, this propensity was not observed in other studies. ${ }^{9}$

Angiopathy, retinopathy, neuropathy and increasing age are considered to represent predisposing factors for onychomycosis in diabetic patients. ${ }^{6,8}$ Impaired sensation in the foot can mask minor trauma and abrasions from nail contact with the skin. This situation together with poor nail grooming, enhance the possibility of microbial infections contributing to the severity of the diabetic foot. ${ }^{8,14}$ Thus, nail infections represent a risk factor in the diabetic patients because of possible sequelae. 
The aim of this work was to revisit the relative prevalence of dermatophyte, yeast and non-dermatophytic mould onychomycoses in type II diabetic patients and in age- and gender-matched non-diabetic controls only suffering from nail alterations.

\section{Materials and methods}

This prospective and comparative study was performed on nail clippings collected for 3 years from 190 type II diabetic outpatients aged from 44 to 57 years. Among them, there were $136(71.6 \%)$ men and $54(28.4 \%)$ women. This group of subjects corresponded to consecutive diabetic patients consulting a dermatology division because they complained from an altered aspect of toenails showing discoloration, thickening and dystrophy. None of the patient had received antifungal therapy for at least the past 6 months. All of them were treated by oral antidiabetic drugs. No information was available about the average control level of their glycaemia. All of them were outpatients who did not report any major clinical consequence of diabetes. A control group was formed by non-diabetic age- and gender-matched subjects presenting similar nail changes. None of the 380 subjects suffered from skin disorders known to alter the nail aspect.

The unfixed nail samples were sliced. Some of the slices were further fragmented and deposited onto Sabouraud medium (Mycoline ${ }^{\circledR}$, BioMerieux, Marcy I'Etoile, France) for mycological cultures. Other slices were embedded in agar for histomycology. After trimming, they were put in contact with Tween-40 in order to soften the nail structure. This material was cut with a microtome for obtaining 6 - $\mu \mathrm{m}$-thick histological sections. They were stained with the periodic acid-Schiff reagents in order to reveal fungi inside the nail plate. The diagnosis of onychomycosis was given when histomycology was positive, and onychodystrophy was considered when this evaluation revealed no fungi. The diagnostic criteria in histomycology have been described in previous studies. ${ }^{17,18}$ In particular finding yeast only was considered to merely represent colonization. By contrast, yeast and pseudofilaments were interpreted as a true infection.

Onychomycoses were further identified according to the nature of the fungus growing at culture. They were thus classified as dermatophyte, yeast or non-dermatophytic mould onychomycoses. When two distinct fungi were seen microscopically and isolated simultaneously from a nail sample, mixed fungal infection of the nail plate was diagnosed. Some onychomycoses remained unidentified because the culture remained negative. This condition most probably indicated the presence of a dead portion of the fungal mycelium in the sampled nail clipping.

Data were expressed as total numbers and percentages. Differences in nail disorder prevalence between diabetic and non-diabetic patients were assessed using the chi-square test and variance analysis. Differences were considered significant when the $P$-value was lower than 0.05 .

\section{Results}

There were no cases of positive cultures for dermatophytes which were negative histologically. By contrast, it was not exceptional to record yeast and moulds growing at culture, without showing nail invasion at histology. This condition corresponding to nail contamination was recorded as onychodystrophy.

Data are presented in Table 1 . When pooling all data irrespective of the gender, the ratio between onychomycoses and non-infectious onychodystrophies reached 1.88 (124 of 66) in diabetic patients, which was twice the value 0.94 (92 of 98) found in non-diabetic controls. A similar finding was obtained when considering each gender group separately. Indeed, the ratio reached 1.97 (90 of 46) in diabetic men and 1.00 (68 of 68 ) in non-diabetic men. It reached 1.70 (34 of 20) in diabetic women and 0.80 (24 of 30) in non-diabetic women.

The proportion of unidentified onychomycoses was not significantly different among the different groups of subjects. In most of these cases, the fungal hyphae were irregular in shape and appeared empty at the histological examination. Among onychomycoses, those caused by dermatophytes, particularly Trichophyton rubrum, prevailed similarly in all groups of individuals. They represented $62.7 \%$ (64 of 102) of the mycologically identified

Table 1 Numbers and percentages of onychomycoses and onychodystrophies in the two age- and sex-matched groups of 190 diabetic and 190 non-diabetic patients.

\begin{tabular}{|c|c|c|c|c|}
\hline \multirow[b]{2}{*}{ Nail alteration } & \multicolumn{2}{|c|}{$\begin{array}{l}\text { Diabetic patients, } \\
n(\%)\end{array}$} & \multicolumn{2}{|c|}{$\begin{array}{l}\text { Non-diabetic patients, } \\
n(\%)\end{array}$} \\
\hline & Men & Women & Men & Women \\
\hline Onychodystrophy & $46(33.8)$ & $20(37.0)$ & $68(50.0)$ & $30(55.6)$ \\
\hline \multicolumn{5}{|l|}{ Onychomycosis } \\
\hline Dermatophyte & $51(37.5)$ & $13(24.1)$ & $39(28.7)$ & $10(18.5)$ \\
\hline Yeast & $8(5.9)$ & $5(9.3)$ & $7(5.1)$ & $3(5.5)$ \\
\hline Mould & $13(9.6)$ & $4(7.4)$ & $8(5.9)$ & $3(5.5)$ \\
\hline Mixed & $6(4.4)$ & $5(9.2)$ & $3(2.2)$ & $3(5.5)$ \\
\hline Unidentified & $12(8.8)$ & $7(13.0)$ & $11(8.1)$ & $5(9.3)$ \\
\hline Total & $136(100)$ & $54(100)$ & $136(100)$ & $54(100)$ \\
\hline
\end{tabular}


diabetic cases and $64.5 \%$ (49 of 76) of the non-diabetic cases. This proportion was higher $(P<0.05)$ in men, either diabetic $(68 \%, 51$ of 75$)$ or non-diabetic $(68.4 \%, 39$ of 57$)$ than in women, either diabetic $(48.1 \%, 13$ of 27$)$ or non-diabetic $(52.6 \%, 10$ of 19$)$.

The prevalence of yeast onychomycoses was similar in diabetic patients $(12.7 \%, 13$ of 102) and in nondiabetic subjects (13.2\%, 10 of 76 ). No gender influence was observed for this type of onychomycosis. Similar prevalence findings were found for non-dermatophytic mould onychomycoses. The identified mould genera encompassed Aspergillus spp., Acremonium spp., Alternaria tennis, Fusarium oxysporum and Scopulariopsis brevicaulis.

\section{Discussion}

In this study, the diagnosis of onychomycosis primarily relied on histomycology. Any positive culture without histological clue for fungal infection was not considered to be sufficient for diagnosing onychomycosis. These stringent criteria are open for discussion because some authors may argue that cultural isolation of a mould from a nail plate with signs of onychodystrophy may indicate the infection. For sure, our diagnostic procedure reduces the risk of overestimating the prevalence of onychomycosis, but it may discretely underestimate it.

Clinically abnormal nails with mycological evidence of fungal disease are not rare in diabetic patients in whom the prevalence of onychomycosis was reported to be higher with increasing age and male gender. ${ }^{9}, 12$ Diabetic men were reported to be about three times as likely to have onychomycosis as women with diabetes. ${ }^{9}$ The present study was based for the first time on the combination of histomycology and cultures for assessing the nature of abnormal nails present in diabetic patients. This dual diagnostic method allows to distinguish with certainty the cases of fungal contamination from fungal invasion of the nail apparatus. This aspect is important to be stressed because the proportion of false-positive and false-negative data at culture alone is high in routine clinical practice. ${ }^{17,18}$

In diabetic patients, men were 2.5 times more numerous than women. This gender difference was present for onychomycoses, but also for fungus-negative onychodystrophies. This indicates that gender-related factors affect the nail structure. This situation has never been reported so far. It may be due to differences in life style, propensity to microtraumatisms, professional activities, sport practices, etc.

The comparison between the present diabetic and non-diabetic groups shows that the proportion of onychomycoses among all nail alterations is much increased in diabetics irrespective of the gender. The increased risk of fungal nail infection was observed for the three families of pathogenic fungi. These findings concern a general group of type II diabetic patients who had no severe clinical manifestations of their metabolic disease.

Dermatophytes, yeast and non-dermatophytic moulds can be responsible for onychomycoses. ${ }^{5,14,17-23}$ In a North American survey in diabetic patients, ${ }^{9}$ the organisms involved in onychomycosis were dermatophytes $(88.2 \%)$, non-dermatophytic moulds $(9.1 \%)$ and Candida spp. (2.7\%). However, other investigators reported that Candida spp. infections of the nail and surrounding area might be more prevalent in diabetic patients. $^{5}$ The present study does not shows any increased prevalence of Candida over other fungi in onychomycoses of diabetic patients. This finding can be opposed to previous observations made in our laboratory showing that $C$. albicans grew more easily on stratum corneum of diabetic patients than on the same structure of non-diabetic subjects. ${ }^{24}$ These older data should now be revisited at the light of the discovery of the innate immune activity of natural antimicrobial peptides synthesized in the epidermis and nails. ${ }^{25-27}$ The antifungal activity of these peptides have not been specifically studied in diabetic subjects so far. Any glycation of these molecules could impair their defence potential against fungi.

While mild toenail onychomycoses may pose little problem to diabetic subjects, more severe, neglected onychomycosis can be a real threat. ${ }^{11}$ The presence of the mycotic nail may result in adjacent nail or skin injury, and provide a reservoir of pathogen fungi, thereby further increasing the risk of serious sequelae and spread of infection. ${ }^{8}$ In particular, high-risk diabetic patients with compromised lower extremities and severe neuropathy were reported to be at increased risk of developing complications from onychomycosis. ${ }^{11}$ Most notably, impaired sensation can make many diabetics less aware of minor abrasions and ulcerations on their feet that may be caused by trauma, form poor nail grooming or by the sharp, brittle of infected nails. ${ }^{8}$ These lesions, in turn, may develop into serious paronychia, cellulitis or other bacterial infections, and contribute to the severity of the diabetic foot. Osteomyelitis can also result from chronically infected nail bed with erosion in diabetic patients because of the close proximity of the nail bed to the underlying bone. ${ }^{11}$ Thus, there is an important clinical rationale for identifying and treating diabetic patients with fungal nail infections. ${ }^{14}$ 
As the population of diabetic subjects keeps growing in western populations, the clinical and pharmacoeconomical impact of onychomycosis in these individuals will likely increase. The increasing onychomycosis incidence, the varied epidemiology and the physical and psychological consequences of the disease emphasize the need for prompt and effective antifungal treatment in susceptible patient populations. Data from clinical trials and postmarketing surveillance suggest that drug interactions with antidiabetic drugs may not be an important issue when itraconazole and terbinafine are administered as antifungals. In contrast, fluconazole has an interaction with some oral antidiabetic agents.

\section{References}

1 Piérard GE. Onychomycosis and other superficial fungal infections of the foot in the elderly. A pan-European survey. Dermatology 2001; 202: 220-4.

2 Macura AB, Macura-Beigun A, Pawlik B. Susceptibility to fungal infections of nails in patients with primary antibody deficiency. Comp Immunol Microbiol Infect Dis 2003; 26: 223-32.

3 Greene RA, Scher RK. Nail changes associated with diabetes mellitus. J Am Acad Dermatol 1987; 16: 1015-21.

4 Evans SL, Nixon BP, Lee I, Lee D, Mooradian AD. The prevalence and nature of podiatric problems in elderly diabetic patients. J Am Geriatr Soc 1991; 39: 241-5.

5 Alteras I, Saryt E. Prevalence of pathogenic fungi in the toe-webs and toe-nails of diabetic patients. Mycopathologia 1979; 16: 157-9.

6 Lugo-Somolinos A, Sanchez JL. Prevalence of dermatophytosis in patients with diabetes. J Am Acad Dermatol 1992; 26: 408-10.

7 Levy LA. Epidemiology of onychomycosis in special risk populations. J Am Pediatr Med Assoc 1997; 87: 546-50.

8 Rich P. Special patient populations: onychomycosis in the diabetic patient. J Am Acad Dermatol 1996; 35: S10-S2.

9 Gupta AK, Konnikov N, MacDonald P et al. Prevalence and epidemiology of toenail onychomycosis in diabetic subjects: a multicentre survey. Br J Dermatol 1998; 139: 665-71.

10 Raboobee N, Aboobaker J, Peer AK. Tinea pedis et unguium in the Muslim community of Durban, South Africa. Int J Dermatol 1998; 37: 759-65.

11 Rich P, Hare A. Onychomycosis in special population. Focus on the diabetic. Int J Dermatol 1999; 38: S17-S9.

12 Rosseeuw D, Katsambas A, Burzykowski T et al. The risk of fungal foot infections in diabetic patients. J Eur Acad Dermatol Venereol 1999; 12: S220.
13 Gupta AK, Gupta MA, Summerbell RC et al. The epidemiology of onychomycosis: possible role of smoking and peripheral arterial disease. J Eur Acad Dermatol Venereol 2000; 14: 466-9.

14 Gupta AK, Humke S. The prevalence and management of onychomycosis in diabetic patients. Eur J Dermatol 2000; 10: $379-84$.

15 Romano C, Massai L, Asta F, Signorini AM. Prevalence of dermatophytic skin and nail infections in diabetic patients. Mycoses 2001; 44: 85-6.

16 Dogra S, Kumar S, Bhansali A, Chakrabarty A. Epidemiology of onychomycosis in patients with diabetes mellitus in India. Int J Dermatol 2002; 41: 647-51.

17 Piérard GE, Arrese JE, Pierre S et al. Diagnostic microscopique des onychomycoses. Ann Dermatol Venereol 1994; 121: 25-9.

18 Piérard GE, Arrese JE, De Doncker P, PiérardFranchimont $\mathrm{C}$. Present and potential diagnostic techniques in onychomycosis. J Am Acad Dermatol 1996; 34: $273-7$.

19 Ginter G, Rieger E, Heigl K, Propst E. Increasing frequency of onychomycosis - is there a change in the spectrum of infectious agents? Mycoses 1996; 39(Suppl. I): 118-22.

20 Greer DL. Evolving role of nondermatophytes in onychomycosis. Int J Dermatol 1997; 34: 521-4.

21 Ghannoum MA, Hajjeh A, Scher R et al. A large-scale North American study of fungal isolates from nails: the frequency of onychomycosis, fungal distribution, and antifungal susceptibility patterns. J Am Acad Dermatol 2000; 43: 641-8.

22 Arrese JE, Piérard GE. Treatment failures and relapses in onychomycosis: a stubborn clinical problem. Dermatology 2003; 207: 255-60.

23 Piérard GE, Arrese JE, Quatresooz P. Histomycologie de la biodiversité des onychomycoses. In: Baran R, Piérard GE (eds), Onychomycoses. Paris, France: Publ. Abrégés Masson, 2004: 91-104.

24 Rurangirwa A, Piérard-Franchimont C, Piérard GE. Growth of Candida albicans on the stratum corneum of diabetic and non-diabetic patients. Mycoses 1990; 33: 253-5.

25 Gallo RL, Murakami M, Ohtake T, Zaiou M. Biology and clinical relevance of naturally occurring antimicrobial peptides. J Allergy Clin Immunol 2002; 110: 823-31.

26 Zasloff M. Antimicrobial peptides of multicellular organisms. Nature 2002; 415: 389-95.

27 Dorschner RA, Lopez-Garcia B, Massie J, Kim C, Gallo RL. Innate immune defense of the nail unit by antimicrobial peptides. J Am Acad Dermatol 2004; 50: 343-8. 